\section{Quantitative Magnetic Resonance Imaging of Satsuma Mandarin Fruit during Growth}

\author{
Christopher J. Clark \\ HortResearch, Palmerston North Research Center, Private Bag 11 030, \\ Palmerston North, New Zealand
}

Annette C. Richardson

HortResearch, Kerikeri Research Center, PO Box 23, Kerikeri, New Zealand

Ken B. Marsh

HortResearch, Mt. Albert Research Center, Private Bag 92 169, Auckland, New Zealand

Additional index words. Citrus unshiu, nondestructive analysis, NMR

\begin{abstract}
Whole-fruit proton magnetic resonance (MR) imaging was performed on satsuma mandarin (Citrus unshiu Markovich cv. Miho Wase) during a 15-week period commencing 10 weeks after anthesis and continuing to maturity, and at 6 weeks after anthesis the following season. Images with long repetition times $(>1600 \mathrm{~ms})$ and short echo times $(20 \mathrm{~ms})$ provided the clearest details of anatomical changes in the peel (flavedo, albedo) and vascular system, while those with similar repetition times but longer echo times $(120 \mathrm{~ms})$ were best for viewing juice sac morphology within pulp segments. At 6 weeks after anthesis, images of fruits of slightly different physiological ages highlighted rapid changes in the vascular bundles and albedo tissue at this stage of development. Variation in the relaxation measurements, $T_{1}$ and $T_{2}$, was determined from quantitative MR images of the juice sacs in equatorial slices, and images of expressed juice from whole fruit. Seasonal measurements of $T_{1}$ determined in situ $(1760 \mathrm{~ms})$ were significantly greater than those in juice (1413 ms). By contrast, there was no mean seasonal difference between in situ $T_{2}$ measurements $(360 \mathrm{~ms})$ and those for juice $(332 \mathrm{~ms})$. No associations between trends in the MR data and total soluble solids, $\mathrm{pH}$, titratable acidity, and sugar and organic acid composition of the juice were established. Cell structure is identified as a hindrance in the use of quantitative MR imaging for probing compositional changes in solution in serial imaging studies.
\end{abstract}

Following introduction of proton magnetic resonance imaging (MRI) in the late 1970s, use of the technique has increased to encompass a range of applications connected with plant materials (Clark et al., 1997; Faust et al., 1997). One development during this time has been Quantitative MRI (QMRI)—a computational procedure based on analysis of a series of image slices whereby the relative contributions that relaxation processes and proton density make to signal intensity in a single image are separated into their component parts (Kuchenbrod et al., 1995). This is especially appealing since it moves MRI away from being a tool that simply takes "pictures" for qualitative analysis, to one in which the calculated images generated by QMRI might be used to detect physiological processes in situ. The rationale for this is 2-fold. Theory suggests that the spin-lattice relaxation time $\left(\mathrm{T}_{1}\right)$, and the spin-spin relaxation time $\left(\mathrm{T}_{2}\right)$, the

Received for publication 18 Feb. 1999. Accepted for publication 6 May 1999. This study was funded by a New Zealand Foundation for Research, Science and Technology contract No. C06620. The cost of publishing this paper was defrayed in part by the payment of page charges. Under postal regulations, this paper therefore must be hereby marked advertisement solely to indicate this fact.

HortSCIEnCE, Vol. 34(6), OCtOBER 1999 published elsewhere, we also report anatomical changes observed in fruit during the course of the study.

\section{Materials and Methods}

Fruit from 6-year-old satsuma mandarin trees growing on Poncirus trifoliata (L.) Raf. rootstock in a commercial orchard in Kerikeri (northern New Zealand) were obtained for imaging. The first samples were harvested 10 weeks after anthesis (6 Nov. 1997) after commencement of Stage II growth (cell enlargement) (Bain, 1958). Further harvests occurred at 16 and 20 weeks, and at 25 weeks when fruit were ripe. Fruit were also harvested again in the following season (6 weeks after anthesis) to examine anatomical features present in immature fruitlets during Stage I growth (cell division).

On each occasion, between 12 and 20 fruit were picked and couriered to the laboratory. At 6,10 , and 16 weeks, the small individual fruits were transported with their peduncles in water to prevent possible moisture loss. Imaging commenced within $24 \mathrm{~h}$ of harvest.

Four fruit were scanned at each harvest date. Thereafter, segments containing the juice sacs from the imaged fruit and their companions were separated from the peel, bulked, and frozen $\left(-20^{\circ} \mathrm{C}\right)$ whole for analysis of juice at a later date. Measurements on centrifuged expressed juice included total soluble solids, $\mathrm{pH}$, titratable acidity, and sugar and organic acid composition after Richardson et al. (1997). Fruit obtained in the second season were not dissected, as too little juice was present at that stage of development for analysis.

Whole-fruit proton magnetic resonance imaging was performed in a 4.7 Tesla widebore (89 mm) magnet (Oxford Instruments, Oxford, England) with custom-designed hardware and software (Eccles et al., 1998). Individual fruit were positioned in either a 40- or 89-mm birdcage coil to image the median equatorial plane using a Hahn spin-echo pulse sequence. Thirteen image slices of this plane were acquired sequentially according to a programmed script. For calculation of the spinlattice relaxation time $\left(T_{1}\right)$, the repetition time $\left(T_{R}\right)$ was varied from 100 to $3200 \mathrm{~ms}$ while the echo time $\left(\mathrm{T}_{\mathrm{E}}\right)$ was held constant $(20 \mathrm{~ms})$. For calculation of the spin-spin relaxation time $\left(\mathrm{T}_{2}\right), \mathrm{T}_{\mathrm{R}}$ was held constant $(3200 \mathrm{~ms})$ while $\mathrm{T}_{\mathrm{E}}$ was varied in 20-ms increments from 20 to 160 ms. Image acquisition ( $n=$ two acquisitions per slice) required $4 \mathrm{~h}$ per sample for a complete set of images. Individual images were acquired as $256 \times 256$ data arrays at fields-ofview ranging from $22 \mathrm{~mm}$ for samples at 6 weeks, through $75 \mathrm{~mm}$ for mature fruit at 25 weeks. Slice thickness was either 1.5 or $3 \mathrm{~mm}$, to achieve acceptable signal : noise ratios. Calculated images of the relaxation parameters $\left(\mathrm{T}_{1}, \mathrm{~T}_{2}\right)$ and pseudo proton spin density were determined by computation as described in Clark et al. (1998).

Relaxation measurements of the juice sac contents at successive times were made on small circular regions-of-interest (ROI) from within the calculated images using the public 
domain program NIH-IMAGE (vers. 1.61available at zippy.nimh.nih.gov). Mean relaxation measurements for each fruit were calculated as the average of six ROI measurements, each in turn the average of relaxation data accumulated over 9 to 100 pixels, the area selected increasing in size as juice sacs enlarged. Areas selected for analysis were identified from the $T_{2}$ maps to avoid inclusion of data from cell walls. Corresponding measurements were subsequently collected from identical locations on $T_{1}$ maps. Statistical analysis was by standard analysis of variance (Genstat 5, Lawes Agricultural Trust, Rothamsted, England). Comparison between the $\mathrm{T}_{1}$ (or $\mathrm{T}_{2}$ ) in fruit vs. expressed juice was analyzed as a single analysis containing the four harvest times. However, because the variances for fruit and juice were very different, a separate analysis was done on each and individual SEDs were calculated to facilitate comparisons within a tissue across time, and between tissues (see Fig. 3).

\section{Results and Discussion}

Flowering in satsuma mandarin takes place over about a 2-week period. Consequently, when young fruitlets were imaged 6 weeks after full bloom, fruit with a range of sizes (and physiological ages) were available. Mandarin are composed of two major morphologically distinct regions - the peel (pericarp) and the edible pulp (endocarp). Within the peel, distinction is made between the external, colored region (epicarp), known as the "flavedo," and the spongy, underlying white-colored tissue (mesocarp), referred to as "albedo" [see Spiegel-Roy and Goldschmidt (1996) for a more detailed description of citrus anatomy]. Dominant features in images $\left(\mathrm{T}_{\mathrm{R}} / \mathrm{T}_{\mathrm{E}}=1600 /\right.$ 20) of the smallest fruits (Fig. 1 a and b) included the flavedo, and individual pulp segments and their accompanying vascular bundles. In transverse sections (Fig. 1b) dorsal bundles were visible arranged on the outside center of each segment, with the ventral vascular bundles forming a ring around the central axis. The bright contrast of the bundles relative to other tissues implied that proton density was high at these locations, the $T_{1}$ relaxation rate was fast (consistent with the presence of concentrated aqueous solution), or possibly both. Less structural detail was evident in the albedo, which, at this point, occupied almost $90 \%$ of the fruit volume. In transverse sections (Fig. 1b), however, two distinct regions plainly existed within this tissue: one consisting of uniform signal intensity extending from the flavedo to midway between the flavedo and pulp segments, the other a region of lower intensity signal surrounding the segments and containing a net of fine vascular elements.

Comparison of images from small fruit (Fig. $1 \mathrm{a}$ and $\mathrm{b}$ ) with those from slightly larger fruit (Fig. $1 \mathrm{c}$ and d) showed several changes occurring within a short time. In the first instance, a faint crescent-shaped shadow was observed on the right-hand edge of certain images (arrowed in Fig. 1d). Displacement of the shadow in the frequency-encoded direc-

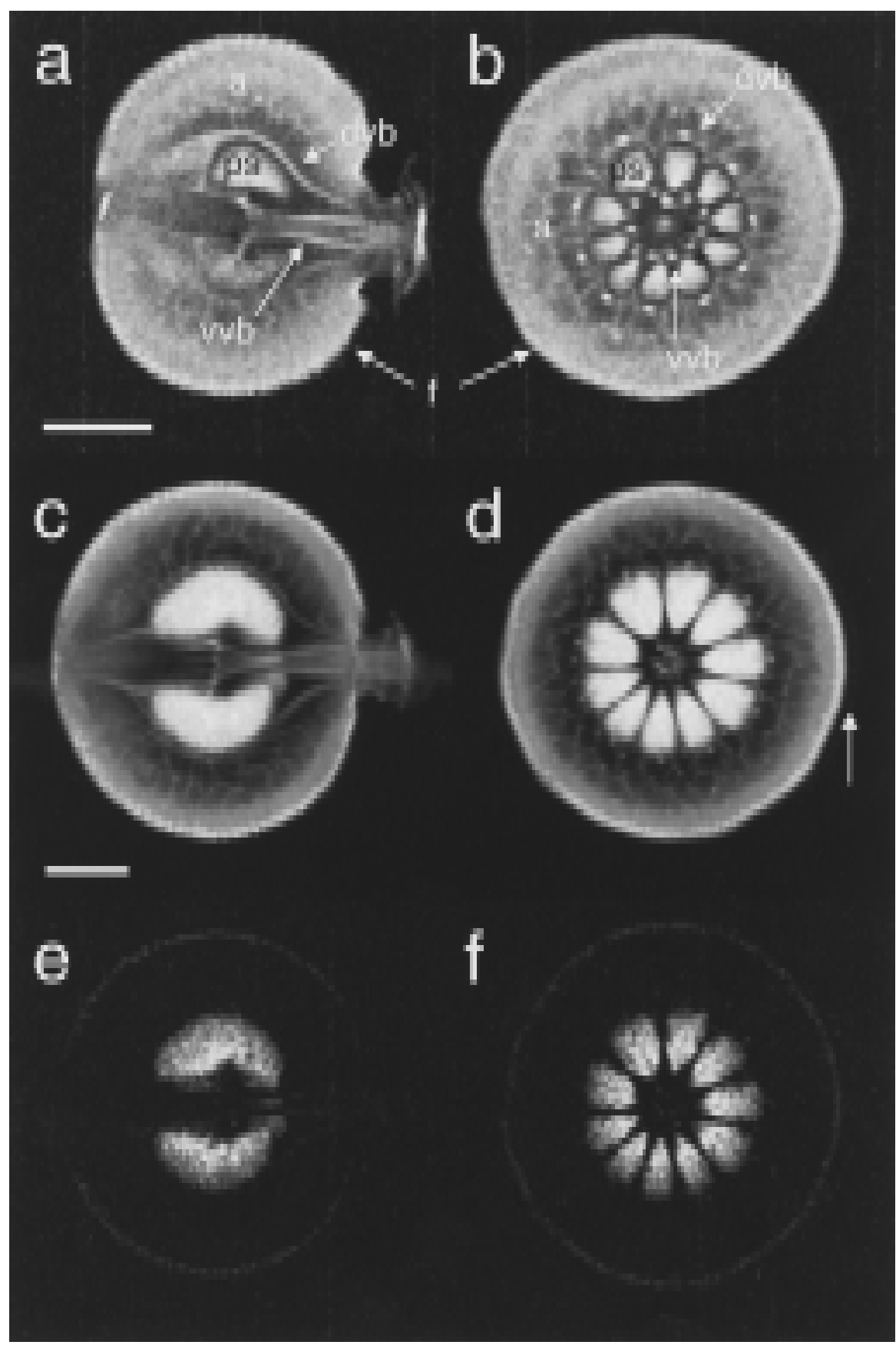

Fig. 1. Proton NMR images of satsuma mandarin during Stage I growth (cell division), 6 weeks after anthesis. Longitudinal (a) and transverse (b) sections of a $17-\mathrm{mm}$-diameter fruitlet $\left(\mathrm{T}_{\mathrm{R}} / \mathrm{T}_{\mathrm{E}}=1600 / 25 \mathrm{~ms}\right.$, $\mathrm{n}=$ four acquisitions, slice thickness $=1.5 \mathrm{~mm}$, in-plane resolution $=85 \mu \mathrm{m})$. Longitudinal $(\mathbf{c}, \mathbf{e})$ and transverse $(\mathbf{d}, \mathbf{f})$ sections of a $22-\mathrm{mm}$-diameter fruitlet. Instrument parameters for $(\mathbf{c})$ and $(\mathbf{d})$ are $\mathrm{T}_{\mathrm{R}} / \mathrm{T}_{\mathrm{E}}$ $=1600 / 25 \mathrm{~ms}, \mathrm{n}=$ eight acquisitions, slice thickness $=3 \mathrm{~mm}$, in-plane resolution $=117 \mu \mathrm{m}$. Instrument parameters for $(\mathbf{e})$ and $(\mathbf{f})$ are $\mathrm{T}_{\mathrm{R}} / \mathrm{T}_{\mathrm{E}}=3200 / 120 \mathrm{~ms}, \mathrm{n}=$ two acquisitions, slice thickness $=3 \mathrm{~mm}$, in-plane resolution $=117 \mathrm{~mm}$. Scale markers are $5 \mathrm{~mm}$. The arrow (panel d) indicates a crescent-shaped shadow (chemical shift artefact) attributed to the presence of essential oils in the flavedo. Abbreviations: albedo (a), dorsal vascular bundles (dvb), flavedo (f), pulp segment (ps), and ventral vascular bundles (vvb).

tion of the image is consistent with a chemical shift artefact, implying that a second protoncontaining species (other than water) was concentrated in the flavedo. The magnitude of the displacement offset is 6 pixels, corresponding to a chemical shift of $1.17 \mathrm{ppm}$. This shift is indicative of the linked methylene groups $\left(\mathrm{CH}_{2}\right)_{n}$ in lipids, which are known to accumulate in multicellular oil glands embedded in the flavedo. Secondly, vascular bundles had become less distinct, especially those in the center of the fruit. Thirdly, pulp segments were noticeably larger, and images obtained with $\mathrm{T}_{\mathrm{R}} / \mathrm{T}_{\mathrm{E}}$ settings of $1600 / 20$ indicated that some structure was present. The morphology of individual juice sacs was seen most clearly, however, in images with longer echo times (e.g., 3200/120, as in Fig. 1 e and f). And lastly, the albedo, which now occupied an estimated $80 \%$ of the fruit volume, was devoid of signal in the region adjacent to the pulp segments exposing the net of fine vascular elements. 
The albedo is a spongy tissue containing large, deeply lobed cells and very large intercellular airspaces that degenerates and disappears during growth (Spiegel-Roy and Goldschmidt, 1996). Analysis of dissected fruit indicated that the albedo was intact at this location. Susceptibility effects (static magnetic fields produced at the boundaries between air- and liquid-filled structures) caused by development of airspaces, or tight binding of water molecules to pectin structures, probably contribute to tissue here having very short $(<20$ ms) $T_{2}$ times. Given that signal intensity of a pixel at any point in the image is proportional to $\mathrm{N}_{\mathrm{H}}\left[1-\exp \left(-\mathrm{T}_{\mathrm{R}} / \mathrm{T}_{1}\right)\right) \exp \left(-\mathrm{T}_{\mathrm{E}} / \mathrm{T}_{2}\right]$, where $\mathrm{N}_{\mathrm{H}}$ is the pseudo proton spin density (closely related to moisture content), a small value for the exponent in the last term can lead to low image intensity. Absence of signal, therefore, does not imply that water (or tissue) was absent at these locations.

During Stage II growth, the calculated images $\left(T_{1}\right.$ and $\left.T_{2}\right)$ of older fruit (Fig. 2) mimicked the $\mathrm{T}_{1}$ - and $\mathrm{T}_{2}$-weighted images of their younger counterparts (cf Fig. $1 \mathrm{~d}$ and f). Details of the albedo, septa membranes separating the pulp segments, and central pith tissue were completely absent during this stage, although albedo extended continuously from the flavedo to the pulp segments until at least 16 weeks after anthesis. In the $T_{1}$ relaxation maps and calculated proton density images (Fig. 2) signal intensity throughout the pulp was essentially uniform. Lack of pronounced change in signal intensity implies that there was no significant change in the radial distribution of moisture content or relaxation properties in equatorial slices, or, if there was, it could not be detected by proton MRI. Definite trends of increasing titratable acidity and decreasing soluble solids from the periphery of the segments toward the core have been reported for other citrus (Ting, 1969), but whether or not this holds for mandarin as well is not known. Gradients in shading were observed in pulp segments of young fruit (Fig. 1f). These probably arise as a result of having to use a 1.5or 3-mm slice thickness and hence averaging two tissues (pulp and albedo) with very different relaxation properties in the outer regions of the pulp segments.

Analysis of discrete regions-of-interest (ROIs) within the calculated maps $\mathrm{T}_{1}$ and $\mathrm{T}_{2}$ (Fig. 2) were used to probe trends in the seasonal relaxation data. In situ measurements of $T_{1}$ fluctuated $\approx 1760 \mathrm{~ms}$ during the experiment, and, on average, were significantly greater $(P<0.001)$ than those in expressed juice $($ mean $=1413 \mathrm{~ms})($ Fig. 3$)$. $\mathrm{T}_{1}$ measurements in expressed juice, on the other hand, increased nearly linearly to a maximum of $1500 \mathrm{~ms}$ at 20 weeks after anthesis. Thus removal of matrix effects by juicing reduced fluctuations in the localized data and led to a uniform seasonal pattern with shorter relaxation times. Shorter relaxation times in juice relative to in situ data can be attributed to a higher solute concentration in the expressed juice, a greater solution viscosity, or the presence of macromolecular species not formerly residing in vacuoles.

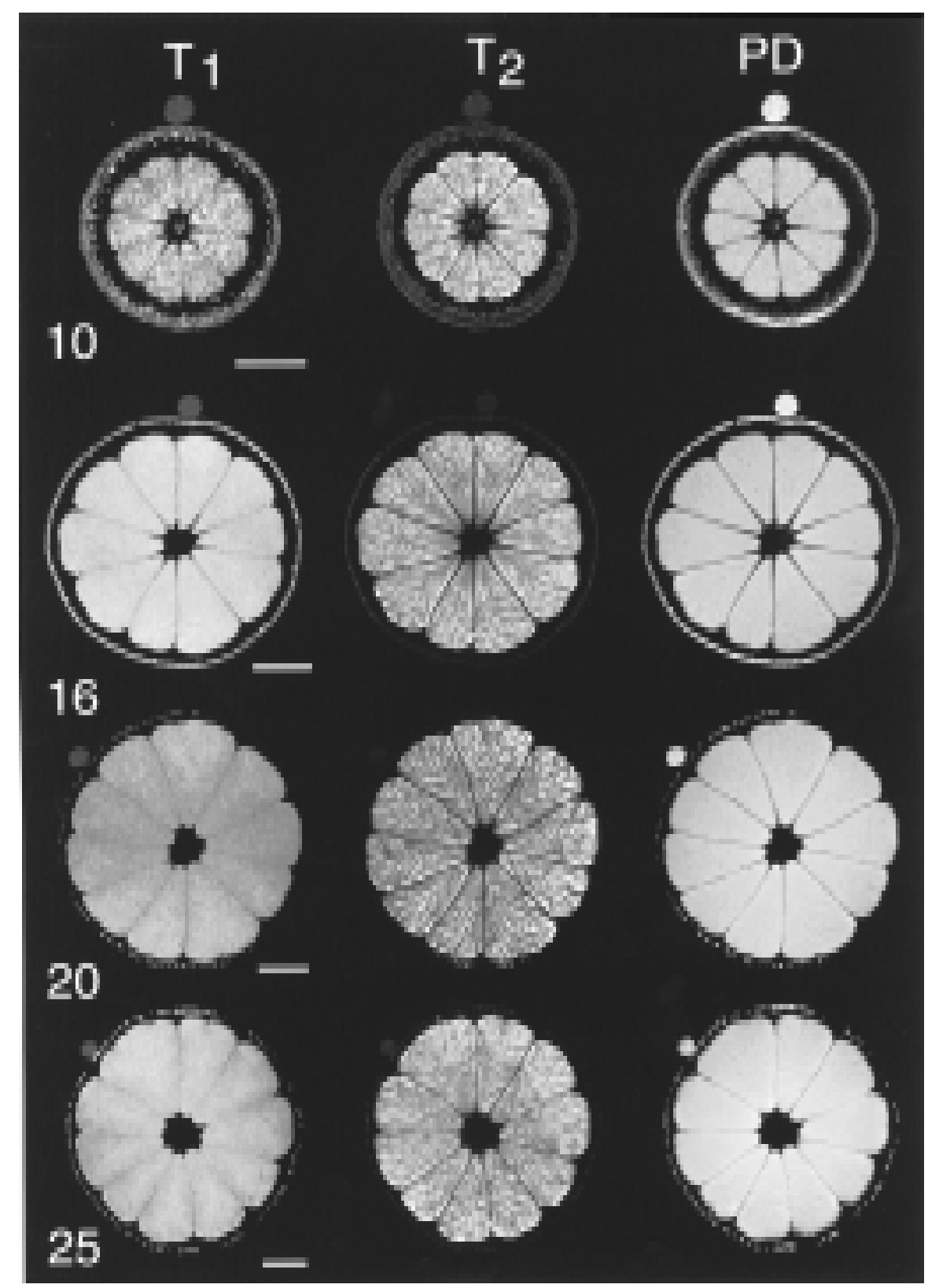

Fig. 2. Quantitative magnetic resonance images of the spin-lattice relaxation time ( $T_{1}$, left-hand column), spin-spin relaxation time $\left(\mathrm{T}_{2}\right.$, middle column) and pseudo proton spin density (right-hand column) for satsuma mandarin fruits during Stage II growth (cell enlargement). The small circular feature in each image is a tube of $200 \mathrm{~mm} \mathrm{MnSO}_{4}$ internal standard solution attached to the outside of the fruit. Numbers indicate weeks after anthesis and the scale marker for each row $=10 \mathrm{~mm}$. In-plane resolutions range from $176 \mu \mathrm{m}$ (Week 10) to $293 \mu \mathrm{m}$ (Week 25). The images are calibrated on a 256-point gray scale, where, in the $\mathrm{T}_{1}$ column, for example, black $=0$ and white $=3000 \mathrm{~ms}$. Calibration scales for the $\mathrm{T}_{2}$ images are 0 to $300 \mathrm{~ms}$, and 0 to $1200 \mathrm{~ms}$ (at 20 weeks).

In contrast, for three of the four harvests there were no significant differences between mean quantitative $\mathrm{T}_{2}$ measurements from juice sacs $(360 \mathrm{~ms})$ and those in juice itself $(332 \mathrm{~ms})$ (Fig. 3). In this instance, removing the cellular matrix produced no overall difference between the two data sets, meaning that in situ measurements reflected trends in juice. However, there was still a significant difference between the two types of $\mathrm{T}_{2}$ measurement at one harvest, 20 weeks after anthesis. The reason for this was not determined.

Changes in composition over time (Table 1) were qualitatively similar to those reported in fruit from the same location in a previous season (Richardson et al., 1997). Here, the total acid concentration (dominated by citrate) was greatest at Week 16 and declined during the remainder of the season, while the total sugar concentration (dominated by sucrose) increased continuously. No obvious statistical associations could be drawn between $T_{1}$ or $T_{2}$, determined by either approach, and any compositional measurement.

The results of this mandarin study reinforce the impression gained with kiwifruit (Clark et al., 1998) that QMRI has limited use for probing solution composition. This is based on the observation that our calculated images were relatively uniform, although quite large 


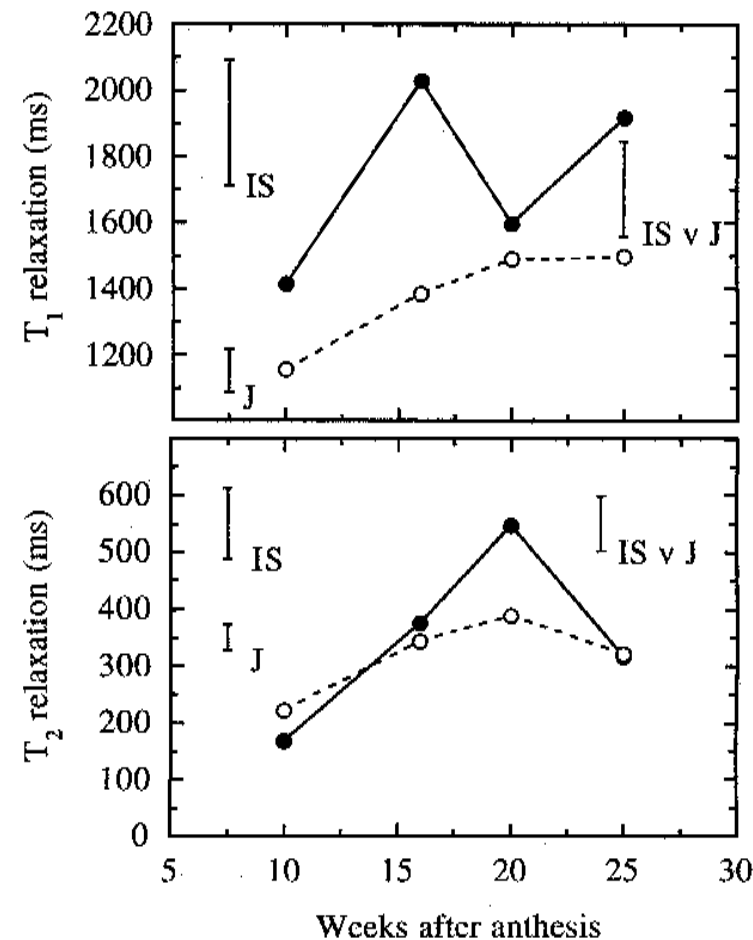

Fig. 3. Changes during growth in spin-lattice $\left(\mathrm{T}_{1}\right)$ relaxation (top) and spin-spin $\left(\mathrm{T}_{2}\right)$ relaxation (bottom panel) in the juice sacs $(\bullet)$ and expressed juice $(\bigcirc)$ of satsuma mandarin. Data points for juice sacs are the means of six ROI measurements on each of four fruit, and the means of four replicates for juice. Error bars are LSDs $(P=0.05)$. Those labeled (IS) and $(J)$ are for between-time comparisons of the in situ and juice data sets, respectively. Those labeled (IS vs. J) are for comparison of in situ and juice means at any particular harvest date.

Table 1. Compositional measurements in expressed juice of satsuma mandarin ('Miho Wase'). Data are means and standard errors of two (at 10 weeks) or five replicates (other sampling times), each containing juice bulked from two or more fruit.

\begin{tabular}{lrrrr}
\hline \hline & \multicolumn{4}{c}{ Weeks after anthesis } \\
\cline { 2 - 5 } & \multicolumn{1}{c}{10} & \multicolumn{1}{c}{16} & \multicolumn{1}{c}{20} & \multicolumn{1}{c}{25} \\
\hline pH & 3.5 & $3.0(0.0)$ & $3.0(0.0)$ & $3.4(0.1)$ \\
Brix (\% soluble solids) & 7.8 & $7.2(0.2)$ & $7.3(0.1)$ & $8.6(0.2)$ \\
Titratable acidity (\%) & 1.8 & $2.6(0.1)$ & $1.7(0.0)$ & $0.9(0.1)$ \\
Sugar : acid ratio & 4.5 & $2.8(0.1)$ & $4.3(0.1)$ & $10.2(0.6)$ \\
Concentration (mg.mL $\left.{ }^{-1}\right)$ & & & & \\
$\quad$ Fructose & 7.3 & $7.3(0.6)$ & $9.3(0.6)$ & $12.4(0.7)$ \\
Glucose & 5.2 & $6.1(0.5)$ & $7.5(0.4)$ & $10.2(0.5)$ \\
Sucrose & 1.4 & $14.3(1.4)$ & $22.4(0.6)$ & $37.2(1.2)$ \\
Total sugars & 13.9 & $27.6(2.3)$ & $39.1(1.3)$ & $60.9(2.3)$ \\
Malate & 1.2 & $1.3(0.1)$ & $1.0(0.1)$ & $0.8(0.1)$ \\
Quinate & 3.0 & $0.8(0.2)$ & $0.6(0.0)$ & $0.3(0.0)$ \\
Citrate & 17.8 & $20.5(1.9)$ & $16.3(0.5)$ & $8.4(0.5)$ \\
Total acids & 22.0 & $23.2(2.0)$ & $17.6(0.6)$ & $9.6(0.6)$ \\
\hline
\end{tabular}

differences in composition between juice sacs within a segment, and between segments in other citrus fruits, have been documented (Goldschmidt and Koch, 1996). Furthermore, our results indicate that differences can exist between in situ measurements averaged over an image slice, and bulk measurements of extracted contents, even under optimal conditions where the sample had large aqueousfilled cells and a single dominant tissue type (the pulp). We attribute these differences to the influence of the sample matrix, which in other circumstances is known to affect NMR parameters (Brownstein and Tarr, 1979; Hills and Duce, 1990). We can therefore anticipate that a sample's physical structure will confound possible linkages between relaxation times and physicochemical measurements when using QMRI. In this instance, however, no useful associations were established even when the structural influences were removed. Clark et al. (1998) discuss the impact on relaxation properties of the concentration of individual components found in fruit solutions. Based on the foregoing, where NMR is used in the future to probe solution composition in fruits, alternative approaches, such as localized spectroscopy (Ishida et al. 1994), solution spectroscopy (Ni and Eads, 1993), chemical shift imaging (Tse et al., 1996), or correlation peak imaging (Ziegler et al., 1996), should be considered.

The motivation for this study was to investigate the usefulness of QMRI. However, it is the images from the immature fruitlets that pose the more intriguing questions. What is the cause of the gradient in signal intensity moving from the flavedo towards the pulp segments? Is it related to morphological or physiological changes in the albedo? At $\approx 6$ weeks after anthesis, the albedo changes from being very dense, with a greenish hue and releasing a white fluid when cut, to a tissue that is uniformly white throughout and less dense. The size of the juice sacs also increased greatly between Fig. $1 \mathrm{~b}$ and d. Does this herald the commencement of juice sac filling and signal the end of Stage I growth? What would even younger fruit have looked like? Can images of fruits this small, or smaller, be obtained with thinner slices? This might shed light on the nature of the vascular connections to the juice sacs and establish whether there are indeed gradients in chemical composition across the segments, or if the central vascular bundles have a role in filling that end of the juice sacs. A previous study (Richardson et al., 1997) has highlighted the importance of the early part of the growth of satsuma mandarin. The results here demonstrate the rapidity with which changes are taking place and suggest that further MRI investigations of young fruit harvested at shorter intervals are warranted.

\section{Literature Cited}

Andrew, E.R. 1980. NMR imaging of intact biological systems. Phil. Trans. Royal Soc. London B289:471-481.

Bain, J.M. 1958. Morphology, anatomical, and physiological changes in the developing fruit of the Valencia orange, Citrus sinensis (L.) Osbeck. Austral. J. Bot. 6:1-28.

Bloembergen, N., E.M. Purcell, and R.V. Pound. 1948. Relaxation effects in nuclear magnetic resonance absorption. Phys. Rev. 73:679-712.

Brownstein, K.R. and C.E. Tarr. 1979. Importance of classical diffusion in NMR studies of water in biological cells. Phys. Rev. A19:2446-2453.

Carver, J.P. and R.E. Richards. 1972. A general twosite solution for the chemical exchange produced dependence of $\mathrm{T}_{2}$ upon the Carr-Purcell pulse separation. J. Magn. Reson. 6:89-105.

Clark, C.J., L.N. Drummond, and J.S. MacFall. 1998. Quantitative magnetic resonance imaging of kiwifruit during growth and ripening. J. Sci. Food Agr. 78:349-358.

Clark, C.J., P.D. Hockings, D.C. Joyce, and R.A Mazucco. 1997. Application of magnetic resonance imaging to pre- and post-harvest studies of fruits and vegetables. Postharvest Biol. Technol. 11:1-21.

Eccles, C.D., C.J. Clark, S.L. Codd, and R. Dykstra. 1998. Construction of an MRI system for horticultural and materials science research, p. 4550. In: ENZCon'98, Proc. 5th Electronics N.Z. Conf., Dunedin.

Faust, M., P.C. Wang, and J. Maas. 1997. The use of magnetic resonance imaging in plant science. Hort. Rev. 20:225-266.

Goldschmidt, E.E. and K.E. Koch. 1996. Citrus, p. 797-823. In: E. Zamski and A.A. Schaffer (eds.). Photoassimilate distribution in plants and crops: Source-sink relationships. Marcel Dekker, New York.

Goodman, B.A., B. Williamson, E.J. Simpson, J.A. Chudek, G. Hunter, and D.A.M. Prior. 1996. High-field NMR microscopic imaging of cultivated strawberry fruit. Magn. Reson. Imaging 14:187-196.

Hills, B.P. and S.L. Duce. 1990. The influence of 
chemical and diffusive exchange on water proton transverse relaxation in plant tissues. Magn. Reson. Imaging 8:321-331.

Hinshaw, W.S., P.A. Bottomley, and G.N. Holland. 1979. A demonstration of the resolution of NMR imaging in biological systems. Experientia 35:1268-1269.

Ishida, N., M. Koizumi, and H. Kano. 1994. Ontogenetic changes in water in cherry tomato fruits measured by nuclear magnetic resonance imaging. Scientia Hort. 57:335-346.

Kuchenbrod,E., A. Haase, R. Benkert, H. Schneider, and U. Zimmermann. 1995. Quantitative NMR microscopy on intact plants. Magn. Reson. Imaging 13:447-455.
MacFall, J.S.,P.E.Pfeffer,D.B. Rolin, J.R. MacFall, and G.A. Johnson. 1992. Observation of the oxygen diffusion barrier in soybean (Glycine max) nodules with magnetic resonance microscopy. Plant Physiol. 100:1691-1697.

Ni, Q.W. and T.M. Eads. 1993. Analysis by proton NMR of changes in liquid-phase and solidphase components during ripening of banana. $\mathrm{J}$. Agr. Food Chem. 41:1035-1040.

Richardson, A.C., K.B. Marsh, and E.A. MacRae. 1997. Temperature effects on satsuma mandarin fruit development. J. Hort. Sci. 72:919-929.

Spiegel-Roy, P. and E.E. Goldschmidt. 1996. Biology of citrus. Cambridge Univ. Press, Cambridge, England.
Ting, S.V. 1969. Distribution of soluble components and quality factors in the edible portion of citrus fruits. J. Amer. Soc. Hort. Sci. 94:515519

Tse, T.Y., R.M. Spanswick, and L.W. Jelinski. 1996. Quantitative evaluation of NMR and MRI methods to measure sucrose concentrations in plants. Protoplasma 194:54-62.

Ziegler, A., A. Metzler, W. Kockenberger, M. Izquierdo, E. Komor, A. Haase, M. Decorps, and M. von Kielin. 1996. Correlation peak imaging. J. Magn. Reson. 112B:141-150.

Zion, B., P. Chen, and M.J. McCarthy. 1995. Nondestructive quality evaluation of fresh prunes by NMR spectroscopy. J. Sci. Food Agr. 67:423-429. 